\title{
S-2HG is an immunometabolite that shapes the T-cell response
}

\author{
Rob A Cairns ${ }^{*, 1}$ and Tak W Mak ${ }^{*, 1}$
}

\author{
Cell Death and Differentiation (2017) 24, 195-196; doi:10.1038/cdd.2016.149; published online 23 December 2016
}

The field of immunometabolism has expanded rapidly over the past several years, and it is now clear that specific alterations to the metabolism of immune cells occur in response to endogenous and exogenous stimuli in order to tailor the biochemistry of cells to their functional requirements and microenvironmental context. ${ }^{1}$ Major findings in T cells have shown that, upon activation, a metabolic response is engaged, resulting in a dramatic increase in glycolysis to support rapid proliferation. Subsequently, during the formation of memory T cells, metabolism shifts away from glycolysis and back towards oxidative phosphorylation fueled by fatty acid oxidation. The mechanisms responsible for this change overlap significantly with the adaptive response to changes in oxygen concentration, but the details are not completely understood.

The two chiral forms of the metabolite 2-hydroxyglutarate, $\mathrm{R}-2 \mathrm{HG}$ and $\mathrm{S}-2 \mathrm{HG}$, are produced in mammalian cells, and are usually maintained at low levels in cells and body fluids via the activity of dedicated dehydrogenase enzymes that convert these compounds to a-ketoglutarate (a-KG). Gain-of-function mutations in isocitrate dehydrogenase 1 and 2 (IDH1 and IDH2) lead to the inappropriate production and accumulation of R-2HG, which is thought to contribute to tumorigenesis in several tissues by altering epigenetic state and metabolism. ${ }^{2}$ The discovery of these mutations has led to a concerted effort to understand the tumorigenic mechanisms underlying $\mathrm{R}-2 \mathrm{HG}$ accumulation. However, little is known about the physiological roles of R-2HG or S-2HG. The ability of these molecules to inhibit a broad class of 2-oxoglutarate-dependent dioxygenases, including key epigenetic regulatory enzymes, suggests that they may have roles in altering the function and fate of specific cells.

It has been shown previously that S-2HG increases upon exposure of cells to hypoxia or in situations where mitochondria are dysfunctional. ${ }^{3-5}$ Under these conditions, S-2HG can be produced by malate dehydrogenase $(\mathrm{MDH})$, lactate dehydrogenase A (LDHA), and even wild-type IDH in certain cell types. These previous studies suggest that accumulation of S-2HG plays a role in the process of hypoxic adaptation by causing epigenetic alterations that are necessary for appropriate reprogramming of metabolism. Interestingly, these previous reports found only a minor role for the HIF transcription factor, often considered the master regulator of the hypoxic response, in mediating the observed increases in S-2HG.

In the current issue of Nature, Tyrakis et al. ${ }^{6}$ report on the physiological role of S-2HG in T cells. T cells display a high degree of metabolic flexibility as their needs change during periods of rapid proliferation and cytolytic activity, and these metabolic changes are required for T-cell function and the formation of durable memory cell populations. In addition, these cells operate in a diverse array of microenvironmental conditions, including the hypoxic regions of solid tumors, healing wounds, and inflamed tissue, placing further demands on their metabolic plasticity. Like previous reports, the authors found that S-2HG levels rise in CD8 T cells in response to hypoxia, but further showed that in vitro T-cell activation leads to an increase in the metabolite, even under normoxic conditions. T-cell activation in combination with hypoxia led to extremely high accumulation of intracellular S-2HG. Unlike previous reports, the increase in S-2HG was dependent on intact HIF1 signaling, as S-2HG levels did not increase in HIF1 knockout $T$ cells upon activation or exposure to hypoxia. The S-2HG produced in these cells appears to be derived primarily from extracellular glutamine, and is produced by the LDHA enzyme, as has been shown for other cell types.

The authors go on to characterize the effects of S-2HG on CD8 T-cell function and fate. By treating cells in vitro with cellpermeable S-2HG, and manipulating endogenously produced S-2HG concentrations by altering expression of the enzyme responsible for converting S-2HG to $a-\mathrm{KG}$ (L2HGDH), they show that the elevation of this metabolite causes a set of transcriptional alterations, cell surface phenotypes, and functional characteristics that are very similar to those observed in memory CD8 $\mathrm{T}$ cells. These alterations are accompanied by epigenetic modifications to DNA and histones characteristic of S-2HG inhibition of epigenetic regulatory enzymes (Figure 1).

The acquisition of memory-like characteristics upon S-2HG treatment prompted the authors to determine the effectiveness of these cells in an adoptive T-cell immunotherapy model. It has been proposed that long-lived memory-like T cells rather than short-lived effectors are important for the success of adoptive T-cell immunotherapy, in which $\mathrm{T}$ cells targeting tumor antigens are infused into patients with malignant disease. Treatment of CD8 T cells in culture with cell-

\footnotetext{
${ }^{1}$ The Campbell Family Institute for Breast Cancer Research at Princess Margaret Cancer Centre, University Health Network, Toronto, ON, Canada M5G 2C1 ${ }^{*}$ Corresponding author: Rob A Cairns or Tak W Mak, The Campbell Family Institute for Breast Cancer Research at Princess Margaret Cancer Centre, University Health Network, Toronto, ON, Canada M5G 2C1. Tel: +1 4169464501 x3058; Fax:+1 416 2042276; E-mail: robc@uhnres.utoronto.ca or tmak@uhnresearch.ca
} 


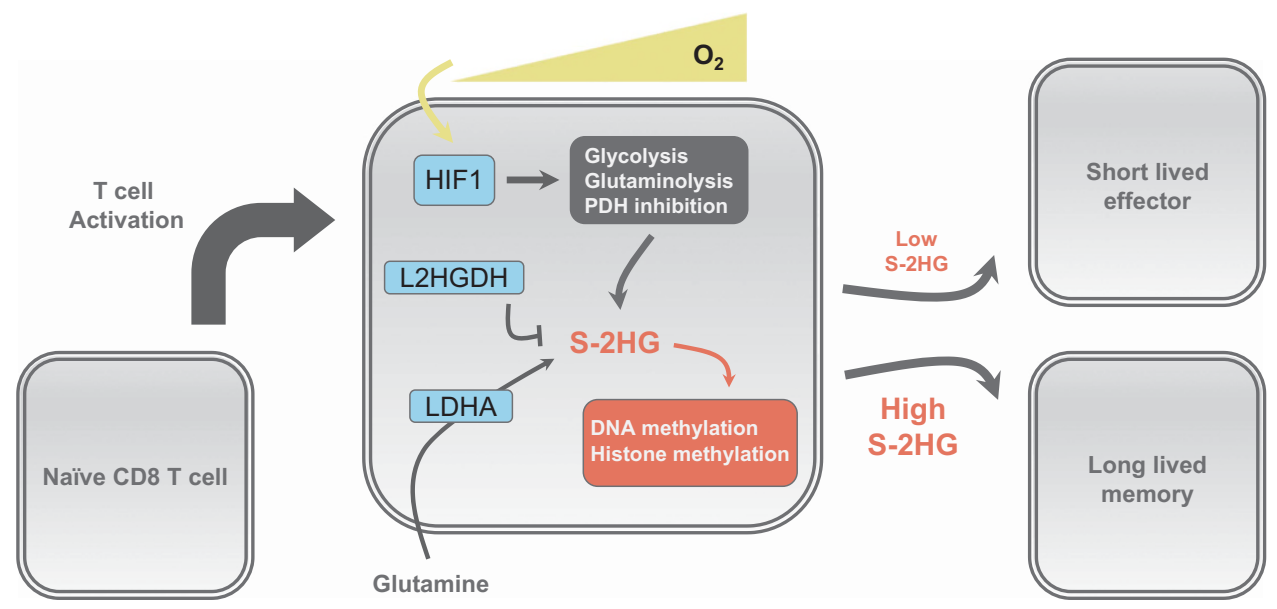

Figure 1 T-cell activation and hypoxia cause metabolic changes, including increased glycolysis, decreased use of glucose in the TCA cycle via inhibition of pyruvate dehydrogenase (PDH), and increased glutamine metabolism. These alterations lead to increased production of S-2HG by LDHA, which overwhelms the capacity of L2HGDH to eliminate it. Increased S-2HG concentrations cause epigenetic alterations that favor the formation of long-lived memory-like CD8 T cells over short-lived rapidly proliferating effectors. S-2HG may act as a immunometabolic messenger, integrating signals from multiple sources to tune the immune response

permeable S-2HG prolonged the survival of these cells when transferred into tumor-bearing hosts, and dramatically increased their anti-tumor activity. This result may indicate a relatively simple method to influence the fate of $\mathrm{T}$ cells prior to adoptive transfer for immunotherapy. Further experiments using additional models and human cells will determine whether this approach will serve to improve this promising therapeutic strategy.

These data suggest that S-2HG may act as a metabolic messenger that integrates signals from a number of sources, including the oxygen level in the microenvironment, cell surface receptor signaling, metabolic state, and perhaps others. Via epigenetic modification, the feedback from S-2HG accumulation may help to fine-tune the immune response. Given that the majority of the work to date has been conducted using in vitro systems, more work is needed to determine how this phenomenon operates in vivo during infection, immunization, and tumorigenesis.
In closing, we would like to join the authors in taking this opportunity to remember the life and work of Dr. Lorenz Poellinger, who passed away during the preparation of this manuscript. Lorenz was an outstanding scientist, a colleague, and a friend who made tremendous contributions to our understanding of the adaptive response to hypoxia, in addition to many other areas of physiology and medicine. He will be greatly missed.

\section{Conflict of Interest}

The authors declare no conflict of interest.
1. O'Neill LAJ et al. Nat Rev Immunol 2016; 16: 553-565.
2. Cairns RA, Mak TW. Cancer Discov 2013; 3: 730-741.
3. Intlekofer AM et al. Cell Metab 2015; 22: 304-311.
4. Oldham WM et al. Cell Metab 2015; 22: 291-303.
5. Wise DR et al. Proc Natl Acad Sci USA 2011; 108: 19611-19616.
6. Tyrakis PA et al. Nature 2016; 540: 236-241. 